\title{
The SMAA-PROMETHEE method
}

\author{
Salvatore Corrente, José Rui Figueira † Salvatore Greco*‡
}

\begin{abstract}
PROMETHEE methods are widely used in Multiple Criteria Decision Aiding (MCDA) to deal with real world decision making problems. In this paper, we propose to apply the Stochastic Multicriteria Acceptability Analysis (SMAA) to the family of PROMETHEE methods in order to explore the whole set of parameters compatible with some preference information provided by the Decision Maker (DM). The application of the presented methodology is described in a didactic example.
\end{abstract}

Keywords: MCDA, PROMETHEE methods, SMAA methods

\section{Introduction}

PROMETHEE is a well-known family of Multiple Criteria Decision Aiding (MCDA) methods (see Figueira et al. 2005a for a collection of surveys on MCDA and Brans and Mareschal 2005, Brans et al. 1984 for a survey on PROMETHEE methods) that deals with several types of real world problems (Behzadian et al., 2010).

In order to apply these methods, the Decision Maker (DM) has to provide some preference information on the parameters involved (preference function shape, weights of criteria and thresholds). This type of preference information can be given directly or indirectly. The DM gives direct preference information when she provides directly all values of the parameters present in the model. The DM supplies indirect preference information when she provides some preferences between alternatives from which compatible preference parameters can be inferred. The indirect preference information requires less cognitive effort from the DM and, for this reason, it is widely used in outranking methods (see for example Dias and Mousseau 2006; Greco et al. 2011; Kadziński et al. 2012; Mousseau and Słowiński 1998; Mousseau et al. 2000).

Recommendations provided by PROMETHEE methods are dependent on the values given to the considered parameters; in fact, generally, different sets of parameters lead to different comparisons between the

\footnotetext{
${ }^{*}$ Department of Economics and Business, University of Catania, Corso Italia 55, 95129 Catania, Italy, e-mails: salvatore.corrente@unict.it, salgreco@unict.it

${ }^{\dagger}$ CEG-IST, Instituto Superior Técnico, Universidade de Lisboa, Av. Rovisco Pais, 1049-001 Lisboa, Portugal, e-mail: figueira@tecnico.ulisboa.pt

${ }^{\ddagger}$ Operations \& Systems Management, Portsmouth Business School, Portsmouth PO1 3DE, United Kingdom
} 
alternatives. For this reason, many methods have been proposed in literature to elicit sets of parameters compatible with some preference information provided by the DM or to get values of the parameters leading to an alternative to occupy the first position(s) in the ranking. Only some of these contributions are cited in the following.

Taking into consideration an MCDA additive method, and therefore obtaining a ranking of the considered alternatives, Mareschal (1988) carries out a sensitivity analysis in order to obtain stability intervals for the criteria weights so that the method provides the same ranking for any combination of the weights inside the said intervals. Sun and Han (2010) solve a linear programming problem to find the most discriminant set of weights compatible with the preference information provided by the DM. Wolters and Mareschal (1995) propose a method to study how the ranking of the alternatives is sensitive to the changes on the weights and to the evaluations of the alternatives, and which modifications on the importance weights are necessary to make a certain alternative the best one. Eppe and De Smet (2012) study the weights that best represent the preferences of the DM and the type of structural information which gives the ranking closest to the ranking provided by the DM. Eppe et al. (2011) use a meta-heuristic in order to induce parameters for the PROMETHEE II model starting from some preference information provided by the DM.

If the DM decides to give indirect preferences, Robust Ordinal Regression (ROR) (Greco et al. 2008; see also Greco et al. 2010 for a survey on ROR) can be applied to explore the whole space of parameters compatible with the preference information provided by the DM. ROR is a family of MCDA methods that simultaneously takes into account all sets of parameters compatible with some preference information provided by the DM considering two preference relations, one possible and one necessary. The necessary preference relation holds between two alternatives $x, y$ if $x$ is at least as good as $y$ for all sets of parameters compatible with the information provided by the DM, while the possible preference relation between two alternatives holds if $x$ is at least as good as $y$ for at least one set of parameters compatible with the preference information provided by the DM. ROR for preferences with interacting criteria modeled through the Choquet integral has been considered in Angilella et al. (2010). ROR has also been applied to the classical PROMETHEE methods in Kadziński et al. (2012) and to the bipolar PROMETHEE methods in Corrente et al. (2014). An axiomatic basis for the concepts of necessary and possible preferences has been given in Giarlotta and Greco (2013).

Similarly to ROR, the Stochastic Multiobjective Acceptability Analysis (SMAA) (Lahdelma et al., 1998; Lahdelma and Salminen, 2001) explores the whole set of parameters compatible with some preference information provided by the DM. SMAA is a family of MCDA methods that takes into account imprecision or lack of data considering probability distributions on the space of criteria weights and on the space of alternatives' evaluations. SMAA has also been applied to dependent uncertainties (Lahdelma and Salminen, 
2002b; Lahdelma et al., 2006) and to preference with interacting criteria (Angilella et al., 2012).

Proposed in this paper is an integrated approach between SMAA and the classical PROMETHEE methods creating the SMAA-PROMETHEE method. On one hand, integrating SMAA and PROMETHEE I method permits computing the frequency of the preference, indifference or incomparability between two alternatives. On the other hand, integrating SMAA and PROMETHEE II method permits to study the different positions an alternative can obtain in the ranking when varying the parameters of the model.

The paper is organized as follows. In the next section the classical PROMETHEE I and II methods are described; in section 3 a description of the SMAA methods introducing their basic concepts is provided; in section 4 the SMAA-PROMETHEE methods and their link with the ROR are presented. Section 5 contains a didactic example and finally some conclusions and future directions of research are presented in the last section.

\section{The classical PROMETHEE methods}

Consider a set of actions or alternatives $A$ evaluated with respect to a set of criteria $G=\left\{g_{1}, \ldots, g_{n}\right\}$, where $g_{j}: A \rightarrow \mathbb{R}, j \in \mathcal{J}=\{1, \ldots, n\}$ and $|A|=m$. PROMETHEE (Brans and Mareschal, 2005; Brans and Vincke, 1985) is a well-known family of MCDA methods that aggregate preference information of a DM through a valued preference relation.

For each criterion $g_{j}$, PROMETHEE methods build a function $P_{j}(x, y)$ representing the degree of preference of $x$ over $y$ on criterion $g_{j}$ and being a non-decreasing function of $d_{j}(x, y)=g_{j}(x)-g_{j}(y)$. The shape of the function for each criterion should be chosen by the analyst in accordance with the DM's preference information. In Brans and Mareschal (2005), six different functions are provided in order to model the preferences of the DM. In the following, we will consider the most used of them, that is, the piecewise linear function:

$$
P_{j}(x, y)=\left\{\begin{array}{lll}
0 & \text { if } \quad d_{j}(x, y) \leq q_{j} \\
\frac{d_{j}(x, y)-q_{j}}{p_{j}-q_{j}} & \text { if } \quad q_{j}<d_{j}(x, y)<p_{j} \\
1 & \text { if } \quad d_{j}(x, y) \geq p_{j}
\end{array}\right.
$$

Notice that the previous function is also known as $V$-Shape criterion in Brans and Mareschal (2005).

Considering for each criterion $g_{j}$ a weight $w_{j}$ (representing the importance of criterion $g_{j}$ within the family of criteria $G$, such that $w_{j} \geq 0$ for all $j$ and $\sum_{j \in \mathcal{J}} w_{j}=1$ ), an indifference threshold $q_{j}$ (being the largest difference $d_{j}(x, y)$ compatible with the indifference between alternatives $x$ and $y$ on criterion $\left.g_{j}\right)$, and a preference threshold $p_{j}$ (being the minimum difference $d_{j}(x, y)$ compatible with the preference of $x$ over $y$ 
on criterion $g_{j}$ ), for each ordered pair of alternatives $(x, y) \in A \times A$, PROMETHEE methods compute

$$
\pi(x, y)=\sum_{j \in \mathcal{J}} w_{j} P_{j}(x, y)
$$

representing how much alternative $x$ is preferred to alternative $y$ taking into account the whole set of criteria. $\pi(x, y)$ can assume values between 0 and 1 and obviously the greater the value of $\pi(x, y)$, the greater the preference of $x$ over $y$.

In order to compare an alternative $x$ with all the other alternatives of the set $A$, PROMETHEE methods consider the negative and the positive flow of $x$ defined in the following way:

$$
\phi^{-}(x)=\frac{1}{m-1} \sum_{z \in A \backslash\{x\}} \pi(z, x) \quad \text { and } \quad \phi^{+}(x)=\frac{1}{m-1} \sum_{z \in A \backslash\{x\}} \pi(x, z) .
$$

$\phi^{-}(x)$ represents how much the alternatives from $A \backslash\{x\}$ are preferred to $x$; the smaller $\phi^{-}(x)$ the better alternative $x$ is; $\phi^{+}(x)$ represents how much $x$ is preferred to the alternatives from $A \backslash\{x\}$; the greater $\phi^{+}(x)$, the better $x$ is. PROMETHEE II also computes the net flow $\phi(x)=\phi^{+}(x)-\phi^{-}(x)$ for each alternative $x \in A$. Taking into account the negative and the positive flows, PROMETHEE I builds a preference $\left(\mathcal{P}^{I}\right)$, an indifference $\left(\mathcal{I}^{I}\right)$ and an incomparability $\left(\mathcal{R}^{I}\right)$ relation on the set of alternatives $A$ while, considering the net flow, PROMETHEE II builds a preference $\left(\mathcal{P}^{I I}\right)$ and an indifference $\left(\mathcal{I}^{I I}\right)$ relation on the set of alternatives $A$ :

$$
\begin{aligned}
& \left\{\begin{array}{l}
x \mathcal{P}^{I} y \text { iff } \Phi^{+}(x) \geq \Phi^{+}(y), \Phi^{-}(x) \leq \Phi^{-}(y) \text { and at least one of the two inequalities is strict } \\
x \mathcal{I}^{I} y \text { iff } \Phi^{+}(x)=\Phi^{+}(y) \text { and } \Phi^{-}(x)=\Phi^{-}(y), \\
x \mathcal{R}^{I} y \text { otherwise. }
\end{array}\right. \\
& \begin{cases}a \mathcal{P}^{I I} b \text { iff } \Phi(a)>\Phi(b), \\
a \mathcal{I}^{I I} b \text { iff } \Phi(a)=\Phi(b) .\end{cases}
\end{aligned}
$$

Let us notice that $\mathcal{P}^{I} \cup \mathcal{I}^{I}$ in PROMETHEE I and $\mathcal{P}^{I I} \cup \mathcal{I}^{I I}$ in PROMETHEE II provide a partial and a complete preorder on the set of alternatives $A$, respectively.

\section{SMAA}

By choosing the decision model and by assigning the values of its parameters, it is straightforward obtaining some results for building further recommendations regarding the problem at hand. Generally, the DM is 
unable to directly provide these parameters or she is unaware of their meaning. Moreover, the evaluations of the considered alternatives can be missing or imprecise in real world decision making problems.

SMAA is a family of MCDA methods that takes into account imprecision or lack of data in the problem at hand considering a probability distribution $f_{W}$ over the space of all compatible weights $W$ and a probability distribution $f_{\chi}$ over the space $\chi \subseteq \mathbb{R}^{m \times n}$ of the alternatives' evaluations $g_{j}(a)$ with $j \in \mathcal{J}$ and $a \in A$ (see Lahdelma et al. 1998, 2003; Lahdelma and Salminen 2001 for the first papers on SMAA, Tervonen and Figueira 2008 for a comprehensive survey on the use of SMAA methods in MCDA and Aertens et al. 2011; Durbach 2009; Lahdelma and Salminen 2009; Menou et al. 2010; Tervonen et al. 2009a,c for some recent publications on SMAA).

Now let us describe some SMAA concepts considering the additive value function, $u\left(x_{i}, w\right)=\sum_{j \in \mathcal{J}} w_{j} g_{j}\left(x_{i}\right)$ with $x_{i} \in A$ and $w \in W$, as preference model.

Without any preference information provided by the DM, the weight space is defined as follows:

$$
\left\{w \in \mathbb{R}^{n}: w_{j} \geq 0, \forall j \in \mathcal{J}, \text { and } \sum_{j \in \mathcal{J}} w_{j}=1\right\} .
$$

If the DM is able to provide some preference information, then the space $W$ is restricted by the constraints translating this preference information. Consequently, with $W$ we shall denote the set of weights compatible with the preference information provided by the DM.

Because $u\left(x_{i}, w\right)$ provides a complete ranking of the alternatives, for each $\xi$ in $\chi$ and $w$ in $W$, SMAA computes the position reached by alternative $x_{i} \in A$ as

$$
\operatorname{rank}(i, \xi, w)=1+\sum_{k \neq i} \rho\left(u\left(\xi_{k}, w\right)>u\left(\xi_{i}, w\right)\right)
$$

where $\rho($ true $)=1$ and $\rho($ false $)=0$. Besides, for each $\xi \in \chi$, SMAA defines the favourable rank weights of alternative $x_{i} \in A$

$$
W_{i}^{r}(\xi)=\{w \in W: \operatorname{rank}(i, \xi, w)=r\}
$$

being the set of possible weights giving to alternative $x_{i}$ the position $r=1, \ldots, m$ in the final rank.

On the basis of the favourable rank weights, SMAA computes the rank acceptability indices, the central weight vectors and the pairwise winning indices.

- The rank acceptability index 


$$
b_{i}^{r}=\int_{\xi \in \chi} f_{\chi}(\xi) \int_{w \in W_{i}^{r}(\xi)} f_{W}(w) d w d \xi
$$

describes the share of parameters giving to alternative $x_{i}$ the position $r$ in the obtained final ranking; in particular, $b_{i}^{1}$ measures the variety of parameters making alternative $x_{i}$ the most preferred. Obviously, the best alternatives are those that have a rank acceptability index greater than zero for the first positions and rank acceptability index close to zero for the lower positions.

- The central weight vector

$$
w_{i}^{c}=\frac{1}{b_{i}^{1}} \int_{\xi \in \chi} f_{\chi}(\xi) \int_{w \in W_{i}^{1}(\xi)} f_{W}(w) w d w d \xi
$$

describes the preferences of a typical DM that makes alternative $x_{i}$ the most preferred.

- The pairwise winning index (Tervonen et al. 2009b and Leskinen et al. 2006)

$$
p_{i k}=\int_{w \in W: \operatorname{rank}(i, \xi, w)>\operatorname{rank}(k, \xi, w)} f_{W}(w) \int_{\xi \in \chi} f_{\chi}(\xi) d \xi d w
$$

is the probability for an alternative $x_{i}$ being more preferred than $x_{k}$.

\section{SMAA-PROMETHEE}

As stated in Section 2, the use of the PROMETHEE methods is based on preference function shape, weights of criteria, indifference and preference thresholds and evaluations of the alternatives on the considered criteria. Obviously, the choice of each of these parameters will influence the results obtained by applying the PROMETHEE methods. In order to take into account the variety of possible results that can be obtained using different values of parameters compatible with the preference information provided by the DM, we shall apply the SMAA methodology. Even if SMAA has already been applied to outranking methods and in particular to ELECTRE methods (Hokkanen et al., 1998; Tervonen et al., 2009a), to the best of our knowledge, applying SMAA to PROMETHEE methods has never been attempted in literature before.

In this section, how to translate different types of preference information provided by the DM and how to obtain results by using the SMAA methododology will be described. 


\subsection{The DM gives information on importance of criteria.}

The DM could be able to give different types of information regarding the importance of criteria. She could order all the criteria with respect to their importance or she could provide an interval of possible values for the same criteria stating, for example, that the importance of criterion $g_{1}$ is between 0.2 and 0.4 or that the importance of criterion $g_{3}$ is between 0.3 and 0.5 and so on.

This preference information can be easily translated by linear inequalities. In the first case, considering $n$ criteria $g_{1}, g_{2}, \ldots, g_{n}$ ordered from the highest to the least important, the corresponding linear inequalities are $w_{1} \geq w_{2} \geq \ldots \geq w_{n}$ while in the second case, interval preferences are translated by $0.2 \leq w_{1} \leq 0.4$ and $0.3 \leq w_{3} \leq 0.5$.

As stated in the previous section, without any preference information provided by the DM, the weight space is defined by $\left\{w \in \mathbb{R}^{n}: w_{j} \geq 0, \forall j \in \mathcal{J}\right.$, and $\left.\sum_{j \in \mathcal{J}} w_{j}=1\right\}$. All the inequalities translating the preference information provided by the DM on the importance of criteria, reduce the weight space defining a polyhedron $W$. Observe that $W$ is a convex set whose points can be obtained as a convex combination of its vertices, $v_{1}, \ldots, v_{k}$. Many papers have provided ways to find such vertices (see for example Solymosi and Dombi 1986; Carrizosa and Conde 1995; Mármol et al. 1998; Puerto et al. 2000). In the easiest case, let us consider $n$ criteria rearranged in a non-increasing order of importance, i.e. $g_{1}$ is at least as important as $g_{2}$ which, in turn, is at least as important as $g_{3}$, and so on. For the Paelinck's theorem (Paelinck, 1974), whose proof is given in Claessens et al. (1991), the vertices of the polyhedron defined by this information are the columns of the following matrix:

$$
M=\left(\begin{array}{ccccc}
1 & \frac{1}{2} & \frac{1}{3} & \cdots & \frac{1}{n} \\
0 & \frac{1}{2} & \frac{1}{3} & \cdots & \frac{1}{n} \\
0 & 0 & \frac{1}{3} & \cdots & \frac{1}{n} \\
\cdots & \cdots & \cdots & \cdots & \cdots \\
0 & 0 & 0 & 0 & \frac{1}{n}
\end{array}\right)
$$

For example, if the DM provides this complete order of criteria $g_{2}, g_{4}, g_{1}, g_{3}$, (i.e. we should have $w_{2} \geq w_{4} \geq$ $\left.w_{1} \geq w_{3}\right)$ the vertices of the corresponding polyhedron are $v_{1}=(0,1,0,0), v_{2}=\left(0, \frac{1}{2}, 0, \frac{1}{2}\right), v_{3}=\left(\frac{1}{3}, \frac{1}{3}, 0, \frac{1}{3}\right)$, and $v_{4}=\left(\frac{1}{4}, \frac{1}{4}, \frac{1}{4}, \frac{1}{4}\right)$.

In this particular case, as the set of weights is convex, a convex combination of said vertices could be used by the analyst to easily get other weights compatible with the preferences provided by the DM.

In order to get robust recommendations for the considered problem, the whole set of compatible weights should be explored and, for this reason, we can apply SMAA and ROR (Greco et al., 2008). ROR is a family 
of MCDA methods that simultaneously take into account all the sets of parameters compatible with the preference information provided by the DM building a necessary and a possible preference relation. Given two alternatives $x$ and $y$, we say that $x$ is necessarily preferred to $y$, and we write $x \succsim^{N} y$, if $x$ is at least as good as $y$ for all compatible sets of parameters, while $x$ is possibly preferred to $y$, and we write $x \succsim^{P} y$, if $x$ is at least as good as $y$ for at least one compatible set of parameters. ROR has already been applied to classical PROMETHEE methods in Kadziński et al. (2012).

Inspired by Corrente et al. (2013), supposing that the weights are the only variables in our model (therefore alternatives' evaluations and thresholds are considered as data of the decision problem), we get the following results on ROR applied to PROMETHEE methods:

Theorem 4.1. Given two alternatives $x$ and $y$, the polyhedron $W$ of weights compatible with the preferences provided by the DM whose vertices are $v_{1}, \ldots, v_{k}$, and denoting by $M_{v_{1}}, \ldots, M_{v_{k}}$ the PROMETHEE models obtained by considering the vectors of weights $v_{1}, \ldots, v_{k}$, respectively, we have for all $x, y \in A$ :

1. $x$ is necessarily preferred to $y$ iff $x$ is at least as good as $y$ for all models $M_{v_{1}}, \ldots, M_{v_{k}}$ (for both PROMETHEE I and II),

2. $x$ is possibly preferred to $y$ iff $x$ is at least as good as $y$ for at least one model among $M_{v_{1}}, \ldots, M_{v_{k}}$ (only for PROMETHEE II).

Proof.

1. It is obvious that if $x$ is necessarily preferred over $y$, then $x$ is at least as good as $y$ for all models $M_{v_{1}}, \ldots, M_{v_{k}}$.

Now, let $M_{v}$ the PROMETHEE model obtained by considering a generic compatible weight vector $v$. Because each weight vector $v$ is a convex combination of the weight vectors $v_{j}$, then there exists $\lambda_{j}$ such that $v=\lambda_{1} v_{1}+\ldots+\lambda_{k} v_{k}$, where $v_{j}=\left(w_{1}^{j}, \ldots, w_{n}^{j}\right)$ and $v=\left(w_{1}^{v}, \ldots, w_{n}^{v}\right)$. Remembering that

$$
\pi^{v}(x, y)=\sum_{l=1}^{n} w_{l}^{v} P_{l}(x, y), \quad w_{l}^{v}=\sum_{j=1}^{k} \lambda_{j} w_{l}^{j} \text { and } \pi^{j}(x, y)=\sum_{l=1}^{n} w_{l}^{j} P_{l}(x, y) \text { for all } j=1, \ldots, k,
$$

then $\pi^{v}(x, y)=\sum_{l=1}^{n}\left(\sum_{j=1}^{k} \lambda_{j} w_{j}^{l}\right) P_{l}(x, y)$ and therefore $\pi^{v}(x, y)=\sum_{j=1}^{k} \lambda_{j} \pi^{j}(x, y)$. We can observe that:

- $\Phi_{v}^{+}(x)=\frac{1}{m-1} \sum_{z \in A \backslash\{x\}} \pi^{v}(x, z)=\frac{1}{m-1} \sum_{z \in A \backslash\{x\}} \sum_{j=1}^{k} \lambda_{j} \pi^{j}(x, z)=\sum_{j=1}^{k} \lambda_{j} \Phi_{j}^{+}(x)$, 


$$
\begin{aligned}
& \text { - } \Phi_{v}^{-}(x)=\frac{1}{m-1} \sum_{z \in A \backslash\{x\}} \pi^{v}(z, x)=\frac{1}{m-1} \sum_{z \in A \backslash\{x\}} \sum_{j=1}^{k} \lambda_{j} \pi^{j}(z, x)=\sum_{j=1}^{k} \lambda_{j} \Phi_{j}^{-}(x), \\
& \text { - } \Phi_{v}(x)=\Phi_{v}^{+}(x)-\Phi_{v}^{-}(x)=\sum_{j=1}^{k} \lambda_{j} \Phi_{j}^{+}(x)-\sum_{j=1}^{k} \lambda_{j} \Phi_{j}^{-}(x)=\sum_{j=1}^{k} \lambda_{j} \Phi_{j}(x) .
\end{aligned}
$$

Because $x$ is at least as good as $y$ for the model $M_{v_{j}}$ if $\Phi_{j}(x) \geq \Phi_{j}(y)$ (or if $\Phi_{j}^{+}(x) \geq \Phi_{j}^{+}(y)$ and $\Phi_{j}^{-}(x) \leq \Phi_{j}^{-}(y)$ using the PROMETHEE I method), it is now straightforward observing that if $\Phi_{j}(x) \geq \Phi_{j}(y)\left(\Phi_{j}^{+}(x) \geq \Phi_{j}^{+}(y)\right.$ and $\left.\Phi_{j}^{-}(x) \leq \Phi_{j}^{-}(y)\right)$ for all $j=1, \ldots, k$ then $\Phi_{v}(x) \geq \Phi_{v}(y)$ $\left(\Phi_{v}^{+}(x) \geq \Phi_{v}^{+}(y)\right.$ and $\left.\Phi_{v}^{-}(x) \leq \Phi_{v}^{-}(y)\right)$.

2. It is obvious that if $x$ is at least as good as $y$ for at least one among $M_{v_{1}}, \ldots, M_{v_{k}}$, then $x$ is possibly preferred to $y$.

Now, let us suppose that $x$ is possibly preferred to $y$. This means that at least one compatible model $M_{v}$ exists such that $\Phi_{v}(x) \geq \Phi_{v}(y)$. Let us suppose, for contradiction, that $x$ is not at least as good as $y$ for all models $M_{v_{1}}, \ldots, M_{v_{k}}$. This means that $\Phi_{j}(x)<\Phi_{j}(y)$ for all $j=1, \ldots, k$. Because $v$ is obtained by convex combination of $v_{1}, \ldots, v_{k}$, then $\lambda_{1}, \ldots, \lambda_{k}$ exist so that $v=\lambda_{1} v_{1}+\ldots+\lambda_{k} v_{k}$, and, as proved previously, $\Phi_{v}(x)=\sum_{j=1}^{k} \Phi_{j}(x)$ for all $x \in A$. Since $\Phi_{j}(x)<\Phi_{j}(y)$ for all $j=1, \ldots, k$ then $\Phi_{v}(a)<\Phi_{v}(b)$ obtaining in this way a contradiction.

We underline that the results obtained by SMAA and ROR are closely linked for the following two reasons: given $x_{i}, x_{k} \in A$,

- if $x_{i}$ is necessarily preferred to $x_{k}$, then the pairwise winning index $p_{i k}=1$,

- if $p_{i k}>0$ then $x_{i}$ is possibly preferred to $x_{k}$.

Let us observe that the viceversa of the two statements above are not true in general. In fact, even if $p_{i k}=1$, other set of parameters not sampled from SMAA for which $x_{k}$ is preferred to $x_{i}$ could exist (and therefore $x_{i}$ is not necessarily preferred to $x_{k}$ ). Analogously, even if $x_{i}$ is possibly preferred to $x_{k}$, then it is still possible that for no compatible set of parameters sampled from SMAA $x_{i}$ is preferred to $x_{k}$ and therefore $p_{i k}=0$.

As SMAA methods compute their indices sampling a large number of sets of parameters among all the compatible ones while the ROR takes into account all the sets of parameters simultaneously, if the sample of the sets of parameters is very high, SMAA results can be used to approximate the results of the necessary and the possible preference relations of the ROR analysis (see Kadziński and Tervonen, 2013a,b for two papers studying the relationships between SMAA and ROR). 


\subsection{The DM gives preference information on thresholds.}

Let us suppose that the analyst, in accordance with the DM, has decided that the V-Shape function (1) is the most appropriate to model her preferences on all criteria. The DM may be interested in giving some preference information on the indifference and preference thresholds for some criterion $g_{j}, j \in \mathcal{J}$ in the form of interval values. That is, she could state that the indifference threshold is a value between $h$ and $k$ or that the preference threshold is lower than a certain value $l$. In this case, indifference and preference thresholds compatible with the preference information provided by the DM should satisfy the following set of constraints

$$
\left\{\begin{array}{l}
q_{j, *} \leq q_{j} \leq q_{j}^{*}, \text { for all } j \in \mathcal{J}, \\
p_{j, *} \leq p_{j} \leq p_{j}^{*}, \text { for all } j \in \mathcal{J}
\end{array}\right.
$$

where $q_{j, *}$, and $q_{j}^{*}$, on one side and $p_{j, *}$ and $p_{j}^{*}$ on the other side are the bounds of the indifference and preference thresholds respectively. Even if, in general, the DM could provide some preferences on the thresholds such that $q_{j}^{*}$ is greater than $p_{j, *}$, for the sake of simplicity we shall suppose that $q_{j}^{*} \leq p_{j, *}$. In the extreme case, in which the DM does not give any information on these bounds, then $q_{j, *}=0, q_{j}^{*}=p_{j, *}=p_{j}$ and $p_{j}^{*}=m_{j}=\max _{x, y \in A}\left|g_{j}(x)-g_{j}(y)\right|$ and therefore the inequalities in (2) become $0 \leq q_{j} \leq p_{j} \leq m_{j}$.

In this case, since the set of constraints (2) defines a convex space, in order to sample other vector of thresholds compatible with the preferences of the DM, one can apply the Hit-And-Run method (Smith, 1984; Tervonen et al., 2013). The Hit-And-Run sampling begins with the choice of one point inside the polyhedron delimited by the constraints translating the preference information provided by the DM. At each iteration, a random direction is sampled from the unit hypersphere that, with the considered position, generates a line. Finally, the sampling of one point inside the segment whose extremes are the intersection of the line with the bounds and the current point is done. Let us point out that the Hit-And-Run sampling method could also be applied in the case in which the DM provides preference information on the importance of criteria because the constraints translating the partial or total order of the importance weights constitute a convex set in the feasible weights space.

\subsection{The evaluations of the alternatives are given imprecisely}

In some decision making problems, evaluations of alternatives are given in an imprecise way. This means that the evaluation of a certain alternative on a particular criterion is not punctual but it could be any value inside an interval. For example, evaluating sport cars, the price of a car could vary between 15000 and 17500 Euros while its maximum speed could vary between $200 \mathrm{~km} / \mathrm{h}$ and $230 \mathrm{~km} / \mathrm{h}$. In this case, for each alternative $x \in A$, one can define its evaluation space as $G(x)=\prod_{j \in \mathcal{J}}\left[\alpha_{j}(x), \beta_{j}(x)\right]$ where $g_{j}(x) \in\left[\alpha_{j}(x), \beta_{j}(x)\right]$ for all 
$j \in \mathcal{J}$ and in particular $\alpha_{j}(x)=\beta_{j}(x)$ if the evaluation of $x$ on criterion $g_{j}$ is given precisely. In this way, the comprehensive evaluation space will be $G=\prod_{x \in A} G(x)$.

In order to take into account such type of preference, before applying the PROMETHEE methods, one should sample a performance table from $G$ and then compute the total or partial ranking of the alternatives.

\subsection{The DM is able to provide some preference information on the considered alter- natives}

In order to get the parameters necessary to apply the methodology, a direct and an indirect techniques have been referred to throughout literature. The DM gives direct preference information when she directly provides all values of the parameters present in the model. The DM supplies indirect preference information when she provides some preferences between alternatives from which compatible preference parameters can be inferred.

Supposing to consider the indirect preference information, the DM could state, for example, that alternative $x$ is preferred to alternative $y$ or that $x$ and $y$ are indifferent. Such preference information can be translated by the following inequalities:

- $\pi(x, y)>\pi(y, x)(\pi(x, y)=\pi(y, x))$ if $x$ is locally preferred (indifferent) to $y$,

- $\Phi(x)>\Phi(y)(\Phi(x)=\Phi(y))$ if $x$ is comprehensively preferred (indifferent) to $y$ and PROMETHEE II is considered,

- $\Phi^{+}(x) \geq \Phi^{+}(y), \Phi^{-}(x) \leq \Phi^{-}(y)$ and $\Phi(x)>\Phi(y)$ if $x$ is comprehensively preferred to $y$ and PROMETHEE I is considered,

- $\Phi^{+}(x)=\Phi^{+}(y)$, and $\Phi^{-}(x)=\Phi^{-}(y)$, if $x$ is comprehensively indifferent to $y$ and PROMETHEE I is considered.

All of the previous constraints are obviously dependent on the weights of criteria, on the indifference and preference thresholds and also on the evaluations of the alternatives. We have to distinguish two cases:

- The evaluations of the alternatives on the considered criteria and the indifference and preference thresholds are punctually defined. In this case, the only variables are the weights of criteria on which eventually the DM could provide some preference information. Therefore, considering the set of constraints translating the preferences of the DM on the weights, one can sample weights compatible with these preferences by using one of the methods introduced in section 4.1. 
- The evaluations of the alternatives are not fixed or the DM wishes to provide some preference information on the thresholds. In this case, the set of constraints modelling the preferences of the DM on the considered alternatives depends on the evaluations of the alternatives and, the problem of sampling a performance table compatible with these preferences is highly not linear. For this reason, one can proceed by sampling a performance table from the evaluation space $G$ and, independently from the performance table sampled, a set of weights and thresholds compatible with the preference information provided by the DM proceeding as described in sections 4.1 and 4.2. Two cases can arise:

- if the sampled parameters (evaluations of the alternatives and thresholds) are compatible with the preferences provided by the DM on the considered alternatives, then these parameters are stored,

- if the sampled parameters are not compatible with the preferences provided by the DM on the considered alternatives, then they are rejected and, if the maximum number of sampling has not yet been reached, a new sampling is performed.

For each sampled set of parameters compatible with the preferences of the DM, one can get:

- preference $P^{I}$, indifference $I^{I}$ and incomparability $R^{I}$ relations by the PROMETHEE I method,

- preference $P^{I I}$ and indifference $I^{I I}$ relations by the PROMETHEE II method,

- ranking of the alternatives by the PROMETHEE II method.

After performing a certain number of iterations, in case of PROMETHEE II one can compute the rank acceptability indices $b_{i}^{r}$ (introduced in section 3), the preference matrix $P$ and the indifference matrix $I$. In particular, the elements in the position $(i, j)$ of the matrices $P$ and $I$ are the global pairwise winning index of alternative $x_{i}$ over $x_{j}$ and the frequency of the indifference between $x_{i}$ and $x_{j}$ respectively. Besides, one can compute the central weight vector $w_{i}^{c}$ and the center of mass $w^{c}$ representing respectively the typical preferences allowing to alternative $x_{i}$ to get the first rank and the average preferences of the DM.

Considering instead the PROMETHEE I method, for each pair of alternatives $\left(x_{i}, x_{j}\right) \in A \times A$ one can compute the global pairwise winning index $p_{i j}$, and the frequency of the indifference or incomparability between $x_{i}$ and $x_{j}$.

As stated previously, PROMETHEE I does not provide a complete ranking of the alternatives but only a partial one. For this reason, we suggest to compute two matrices UP and DOWN of dimension $n \times n$ which elements have the following interpretation: 
- $\mathrm{UP}(i, k)$ is the frequency with which alternative $x_{i}$ is preferred to $k$ other alternatives in $A$ by using the PROMETHEE I method. For example, if $\operatorname{UP}(i, 0)=50 \%$ then one can state that in the $50 \%$ of the simulations, alternative $x_{i}$ is not preferred to any other alternative in $A$. Analogously, if $\mathrm{UP}(i, n-1)=100 \%$, then in all cases alternative $x_{i}$ is preferred to all other alternatives in $A$ by using the PROMETHEE I method.

Consequently, it can be observed that the best alternatives are those one having high frequency for $k=\left\lfloor\frac{n}{2}\right\rfloor, \ldots, n-1$ (the symbol $\lfloor\alpha\rfloor$ denotes the minimum integer contained in $\alpha$ ).

- $\operatorname{DOWN}(i, k)$ represents the frequency with which $k$ other alternatives in $A$ are preferred to alternative $x_{i}$ by using the PROMETHEE I method. For example, if $\operatorname{DOWN}(i, 0)=30 \%$ then it can be stated that in $30 \%$ of the simulations, no alternative is preferred to $x_{i}$. Analogously, if $\operatorname{DOWN}(i, n-1)=100 \%$, then in all cases all alternatives are preferred to $x_{i}$ by using the PROMETHEE I method.

Differently from the previous case, it can be observed that the best alternatives are those which have a high frequency for $k=0, \ldots,\left\lfloor\frac{n}{2}\right\rfloor$.

Observe that the two matrices UP and DOWN can be computed in any MCDA method providing a partial preorder on the set of alternatives $A$ as, for example, ELECTRE II, III and IV (see Figueira et al., 2005b).

Let us also observe that the two matrices UP and DOWN could be computed for the PROMETHEE II method and for each other method providing a complete order of the alternatives in $A$ observing what follows:

- for each $(i, k)$ we will have $\operatorname{UP}(i, k)+\operatorname{DOWN}(i, n-1-k)=100 \%$ because if $x_{i}$ is preferred to $k$ other alternatives, then $n-1-k$ other alternatives are preferred to $x_{i}$. We said " generally"' because in some particular cases $x_{i}$ could be indifferent to other alternatives $x_{j}$ and therefore neither $x_{i}$ is preferred to $x_{k}$ nor $x_{k}$ is preferred to $x_{i}$,

- for each $x_{k} \in A$ and $r=1, \ldots, n, b_{k}^{r}=\mathrm{UP}(k, n-r)$ and $b_{k}^{r}=\operatorname{DOWN}(k, r-1)$. In fact, if $x_{k}$ is in position $r$ with a certain frequency, then with the same frequency $x_{k}$ is preferred to $n-r$ alternatives and, $r-1$ alternatives are preferred to $x_{k}$.

The global pairwise winning index $p_{i j}$ is computed comparing $x_{i}$ and $x_{j}$ but taking into account all the alternatives in the set $A$. In order to compare alternatives $x_{i}$ and $x_{j}$ without taking into account all the other alternatives, one can compute the local pairwise winning index being the frequency with which $\pi\left(x_{i}, x_{j}\right)>\pi\left(x_{j}, x_{i}\right)$ that is the frequency with which $x_{i}$ is preferred to $x_{j}$ more than $x_{j}$ is preferred to $x_{i}$. 


\section{Illustrative example}

In this section we shall apply SMAA to the classical PROMETHEE methods to deal with a multiple criteria decision making problem in which a DM has to choose among five cars evaluated on four different criteria: Price, Acceleration, Maximum speed and Consumption. The evaluations of the five cars on the four criteria are reported in Table 1.

Table 1: Performance Table

\begin{tabular}{|c|c|c|c|c|c|}
\hline & Cars & $\begin{array}{c}\text { Price } \\
\text { Euro }\end{array}$ & $\begin{array}{c}\text { Acceleration } \\
\mathbf{0} / \mathbf{1 0 0} \mathbf{~ k m} / \mathbf{h}\end{array}$ & $\begin{array}{c}\text { Max speed } \\
\mathbf{k m} / \mathbf{h}\end{array}$ & $\begin{array}{c}\text { Consumption } \\
\mathbf{l} / \mathbf{1 0 0} \mathbf{k m}\end{array}$ \\
\hline (PE) & PEUGEOT 208 1.6 8V & {$[16000,18000]$} & {$[9.5,11.5]$} & {$[175,190]$} & {$[2.8,4.5]$} \\
& e-HDi 92 CV Stop\&Start 3p. Allure & & & & \\
\hline (CI) & $\begin{array}{c}\text { CITROEN C3 } \\
\text { 1.4 HDi 70 Seduction }\end{array}$ & {$[15000,16500]$} & {$[12.7,14.2]$} & {$[155,170]$} & {$[3,4.6]$} \\
\hline (FI) & $\begin{array}{c}\text { FIAT 500 0.9 } \\
\text { TwinAir Turbo Street }\end{array}$ & {$[14500,15800]$} & {$[10,12]$} & {$[165,180]$} & {$[3,5]$} \\
\hline (SK) & $\begin{array}{l}\text { SKODA Fabia 1.2 } \\
\text { TDI CR 75 CV 5p. GreenLine }\end{array}$ & {$[14100,15650]$} & {$[13.2,15.2]$} & {$[160,181]$} & {$[2.5,4.3]$} \\
\hline (LA) & $\begin{array}{l}\text { LANCIA Ypsilon 5p } \\
\text { 1.3 MJ 95 CV 5p. S\&S Gold }\end{array}$ & {$[15500,17100]$} & {$[10.6,12.8]$} & {$[175,191]$} & {$[3.2,4.4]$} \\
\hline
\end{tabular}

Let us suppose that the DM in accordance with the analyst decides that the V-shape function represents her preferences and she arranges the criteria in a non-increasing order of preference as follows: Price, Consumption, Acceleration and Maximum speed. This means that, denoting with $w_{P}, w_{A}, w_{M}$ and $w_{C}$ the weights of the four criteria, the DM preferences are translated by the constraints $w_{P} \geq w_{C} \geq w_{A} \geq w_{M}$. Regarding the indifference and the preference thresholds, the DM gives the information reported in Table 2 .

Table 2: Indifference and preference thresholds

\begin{tabular}{|c|c|c|c|c|}
\hline Thresholds & $\begin{array}{c}\text { Price } \\
\text { Euro }\end{array}$ & $\begin{array}{c}\text { Acceleration } \\
\mathbf{0} / \mathbf{1 0 0} \mathbf{~} \mathbf{m} / \mathbf{h}\end{array}$ & $\begin{array}{c}\text { Max speed } \\
\mathbf{~ k m} / \mathbf{h}\end{array}$ & $\begin{array}{c}\text { Consumption } \\
\mathbf{l} / \mathbf{1 0 0} \mathbf{k m}\end{array}$ \\
\hline $\mathbf{q}_{\mathbf{j}}$ & {$[500,1000]$} & {$[2,3]$} & 30 & {$[0,0.5]$} \\
\hline $\mathbf{p}_{\mathbf{j}}$ & {$[1500,2000]$} & {$[3,5]$} & 40 & 0.5 \\
\hline
\end{tabular}

Since the DM is interested in getting a general overview of the five cars, we present her with the results in Tables 3 and 4 achieved by applying SMAA to the classical PROMETHEE I and II methods.

According to Tervonen and Lahdelma (2007), in order to achieve error limits of 0.01 for $b_{i}^{r}$, we sampled 10000 sets of parameters compatible with the preference information provided by the DM. The results show that (SK) is almost surely the best car. Indeed, looking at the positive and the negative flows separately, that is taking into account how much an alternative is preferred to all other alternatives and how much all 
other alternatives are preferred to it, we obtain:

- (SK) is preferred to each other car with a frequency of at least $77 \%$ (see the fourth row of Table 3(a)),

- (SK) is preferred to all other cars with a frequency of $66 \%$ (see the fourth row of Table 3(b)),

- the frequency that none of the other alternatives is preferred to $(\mathbf{S K})$ is $83 \%$ (see the fourth row of Table 3(c)).

In the same way, (PE) should be the worst car because:

- Each car is preferred to (PE) with a frequency of at least $49 \%$ (see the first column of Table 3(a)),

- $(\mathbf{P E})$ is preferred to no other car with a frequency of $71 \%$ (see the first row of Table 3(b)).

- All other cars are preferred to (PE) with a frequency of $44 \%$ (see the first row of Table 3(c)),

Looking at the second best car, it seems that the choice should be between CI and FI because both of them are more globally preferred to SK (see fourth column of Table 3(a)) and more locally preferred to SK (see fourth column of Table $3(\mathrm{~d})$ ).

Table 3: Results obtained by applying SMAA to the classical PROMETHEE I method

(b) $\mathrm{UP}(i, k)$ : frequency with which al-

(a) Global pairwise winning indices (in ternative $x_{i}$ is preferred to $k$ other alpercentage)

\begin{tabular}{cccccc}
\hline & PE & CI & FI & SK & LA \\
\hline PE & 0 & 9 & 9 & 1 & 22 \\
CI & 70 & 0 & 38 & 9 & 65 \\
FI & 75 & 43 & 0 & 9 & 64 \\
SK & 94 & 78 & 77 & 0 & 92 \\
LA & 49 & 21 & 19 & 3 & 0 \\
\hline
\end{tabular}
ternatives (in percentage)

\begin{tabular}{cccccc}
\hline UP & $\mathbf{0}$ & $\mathbf{1}$ & $\mathbf{2}$ & $\mathbf{3}$ & $\mathbf{4}$ \\
\hline PE & 71 & 19 & 7 & 2 & 0 \\
CI & 14 & 25 & 32 & 23 & 6 \\
FI & 14 & 23 & 27 & 29 & 7 \\
SK & 1 & 4 & 10 & 19 & 66 \\
LA & 37 & 38 & 17 & 7 & 1 \\
\hline
\end{tabular}

(c) $\operatorname{DOWN}(i, k)$ : frequency with which $k$ alternatives are preferred to alternative $x_{i}$ (in percentage)

\begin{tabular}{cccccc}
\hline DOWN & $\mathbf{0}$ & $\mathbf{1}$ & $\mathbf{2}$ & $\mathbf{3}$ & $\mathbf{4}$ \\
\hline PE & 3 & 12 & 18 & 24 & 44 \\
CI & 14 & 37 & 30 & 14 & 4 \\
FI & 18 & 41 & 24 & 13 & 4 \\
SK & 83 & 13 & 4 & 1 & 0 \\
LA & 4 & 17 & 27 & 36 & 16
\end{tabular}

(d) Local pairwise winning index (in percentage)

\begin{tabular}{cccccc}
\hline & PE & CI & FI & SK & LA \\
\hline PE & 0 & 19 & 12 & 2 & 33 \\
CI & 79 & 0 & 49 & 13 & 56 \\
FI & 86 & 44 & 0 & 13 & 66 \\
SK & 98 & 77 & 84 & 0 & 92 \\
LA & 54 & 30 & 27 & 6 & 0 \\
\hline
\end{tabular}

Because the DM wants to be as cautious as possible in choosing the best car, she would like to have more information about the possible rankings that can be obtained varying the different parameters and about which preferences make each alternative the best. For this reason, we decided to show her the results 
obtained by applying SMAA to the classical PROMETHEE II method too. We point out that looking at the first two columns of Table 4(a), that is at the frequency by which a car reached the first two positions, (FI) is slightly better than $(\mathbf{C I})$ while it is still confirmed that $\mathbf{P E}$ is the worst car.

Table 4: Results obtained by applying SMAA to the classical PROMETHEE II method

(a) Rank acceptability indices (in percentage)

\begin{tabular}{cccccc}
\hline & $\mathbf{b}_{\mathbf{j}}^{\mathbf{1}}$ & $\mathbf{b}_{\mathbf{j}}^{\mathbf{2}}$ & $\mathbf{b}_{\mathbf{j}}^{\mathbf{3}}$ & $\mathbf{b}_{\mathbf{j}}^{\mathbf{4}}$ & $\mathbf{b}_{\mathbf{j}}^{\mathbf{5}}$ \\
\hline $\mathbf{P E}$ & 1 & 5 & 12 & 23 & 60 \\
$\mathbf{C I}$ & 9 & 31 & 32 & 20 & 8 \\
$\mathbf{F I}$ & 12 & 36 & 26 & 18 & 8 \\
$\mathbf{S K}$ & 76 & 16 & 6 & 2 & 0 \\
$\mathbf{L A}$ & 2 & 12 & 24 & 38 & 25 \\
\hline
\end{tabular}

(b) Central weight vectors

\begin{tabular}{ccccc}
\hline Cars & $\mathbf{w}_{\mathbf{P}}$ & $\mathbf{w}_{\mathbf{A}}$ & $\mathbf{w}_{\mathbf{M}}$ & $\mathbf{w}_{\mathbf{C}}$ \\
\hline $\mathbf{P E}$ & 0.46 & 0.14 & 0.06 & 0.33 \\
$\mathbf{C I}$ & 0.49 & 0.15 & 0.06 & 0.29 \\
FI & 0.54 & 0.13 & 0.05 & 0.26 \\
SK & 0.52 & 0.14 & 0.06 & 0.26 \\
$\mathbf{L A}$ & 0.49 & 0.13 & 0.06 & 0.30 \\
\hline
\end{tabular}

(c) Center of mass

\begin{tabular}{cccc}
\hline $\mathbf{w}_{\mathbf{P}}$ & $\mathbf{w}_{\mathbf{A}}$ & $\mathbf{w}_{\mathbf{M}}$ & $\mathbf{w}_{\mathbf{C}}$ \\
\hline 0.52 & 0.14 & 0.06 & 0.27 \\
\hline
\end{tabular}

From Table 4(a) we see that every car could fill the first position in the final rank while from Table 4(b) we understand that criterion Maximum speed has a general marginal importance in the final decision. In particular, it can be surprising to see that $(\mathbf{P E})$ could achieve the first position, and therefore asking for which values of the parameters it could happen.

Table 5: Average evaluations of the alternatives and of the thresholds for which (PE) gets the first position

\begin{tabular}{ccccc}
\hline & $\begin{array}{c}\text { Price } \\
\text { Euro }\end{array}$ & $\begin{array}{c}\text { Acceleration } \\
\mathbf{0 / 1 0 0 ~} \mathbf{~ k m} / \mathbf{h}\end{array}$ & $\begin{array}{c}\text { Max speed } \\
\mathbf{k m} / \mathbf{h}\end{array}$ & $\begin{array}{c}\text { Consumption } \\
\text { l/100km }\end{array}$ \\
\hline $\mathbf{P E}$ & 16294.6 & 10.57 & 183.35 & 3.09 \\
$\mathbf{C I}$ & 15801.4 & 13.39 & 162.85 & 3.92 \\
$\mathbf{F I}$ & 15229.3 & 11.08 & 172.53 & 4.15 \\
$\mathbf{S K}$ & 15244.7 & 14.09 & 170.21 & 3.89 \\
$\mathbf{L A}$ & 16168.6 & 11.77 & 183.87 & 3.84 \\
\hline & & & & \\
\hline $\mathbf{q}_{\mathbf{j}}$ & 772.75 & 2.52 & 30 & 0.22 \\
$\mathbf{p}_{\mathbf{j}}$ & 1778.57 & 4.02 & 40 & 0.5 \\
\hline
\end{tabular}

Looking at Table 5 we can observe that even if $(\mathbf{P E})$ has the highest price among the five considered cars, and that the price is the most important criterion for the DM, it has the lowest consumption and this criterion is the second most important and has an importance weight equal to 0.27. 
In Table 6 we reported the evaluations and the thresholds for which the other four alternatives could reach the first position.

Table 6: Average evaluations and thresholds for which each alternative reached the first position

\section{(a) CITROEN C3}

\begin{tabular}{ccccc}
\hline & $\begin{array}{c}\text { Price } \\
\text { Euro }\end{array}$ & $\begin{array}{c}\text { Acceleration } \\
\mathbf{0 / 1 0 0} \mathbf{~ k m} / \mathbf{h}\end{array}$ & $\begin{array}{c}\text { Max speed } \\
\mathbf{k m} / \mathbf{h}\end{array}$ & $\begin{array}{c}\text { Consumption } \\
\mathbf{l} / \mathbf{1 0 0 \mathbf { k m }}\end{array}$ \\
\hline $\mathbf{P E}$ & 17068.2 & 10.49 & 182.31 & 3.65 \\
$\mathbf{C I}$ & 15500.1 & 13.50 & 162.25 & 3.31 \\
$\mathbf{F I}$ & 15203.5 & 10.96 & 172.53 & 4.14 \\
$\mathbf{S K}$ & 15068.4 & 14.16 & 170.56 & 3.89 \\
$\mathbf{L A}$ & 16319.3 & 11.67 & 183.00 & 3.79 \\
\hline & & & & \\
\hline $\mathbf{q}_{\mathbf{j}}$ & 758.80 & 2.51 & 30 & 0.24 \\
$\mathbf{p}_{\mathbf{j}}$ & 1757.99 & 3.98 & 40 & 0.5 \\
\hline
\end{tabular}

(c) SKODA Fabia 1.2

\begin{tabular}{ccccc}
\hline & $\begin{array}{c}\text { Price } \\
\text { Euro }\end{array}$ & $\begin{array}{c}\text { Acceleration } \\
\mathbf{0 / 1 0 0 ~} \mathbf{~ k m} / \mathbf{h}\end{array}$ & $\begin{array}{c}\text { Max speed } \\
\mathbf{k m} / \mathbf{h}\end{array}$ & $\begin{array}{c}\text { Consumption } \\
\text { l/100km }\end{array}$ \\
\hline $\mathbf{P E}$ & 16998.9 & 10.50 & 182.51 & 3.65 \\
$\mathbf{C I}$ & 15776 & 13.44 & 162.50 & 3.84 \\
$\mathbf{F I}$ & 15169.6 & 10.99 & 172.52 & 4.07 \\
$\mathbf{S K}$ & 14816.2 & 14.21 & 170.60 & 3.25 \\
$\mathbf{L A}$ & 16298.3 & 11.69 & 182.98 & 3.80 \\
\hline & & & & \\
\hline $\mathbf{q}_{\mathbf{j}}$ & 747.97 & 2.50 & 30 & 0.25 \\
$\mathbf{p}_{\mathbf{j}}$ & 1747.43 & 3.98 & 40 & 0.5 \\
\hline
\end{tabular}

(b) FIAT 5000.9

\begin{tabular}{ccccc}
\hline & $\begin{array}{c}\text { Price } \\
\text { Euro }\end{array}$ & $\begin{array}{c}\text { Acceleration } \\
\mathbf{0 / 1 0 0} \mathbf{~ k m} / \mathbf{h}\end{array}$ & $\begin{array}{c}\text { Max speed } \\
\mathbf{k m} / \mathbf{h}\end{array}$ & $\begin{array}{c}\text { Consumption } \\
\mathbf{1 / 1 0 0 \mathbf { m }}\end{array}$ \\
\hline $\mathbf{P E}$ & 16970.8 & 10.54 & 182.44 & 3.66 \\
$\mathbf{C I}$ & 15814.4 & 13.43 & 162.45 & 3.85 \\
$\mathbf{F I}$ & 14956.4 & 11.08 & 172.39 & 3.41 \\
$\mathbf{S K}$ & 15079.1 & 14.11 & 170.29 & 3.83 \\
$\mathbf{L A}$ & 16308.6 & 11.70 & 182.98 & 3.78 \\
\hline & & & & \\
\hline $\mathbf{q}_{\mathbf{j}}$ & 747.52 & 2.50 & 30 & 0.24 \\
$\mathbf{p}_{\mathbf{j}}$ & 1753.39 & 4.06 & 40 & 0.5 \\
\hline
\end{tabular}

(d) LANCIA Ypsilon 5p

\begin{tabular}{ccccc}
\hline & $\begin{array}{c}\text { Price } \\
\text { Euro }\end{array}$ & $\begin{array}{c}\text { Acceleration } \\
\mathbf{0 / 1 0 0} \mathbf{~ k m} / \mathbf{h}\end{array}$ & $\begin{array}{c}\text { Max speed } \\
\mathbf{k m} / \mathbf{h}\end{array}$ & $\begin{array}{c}\text { Consumption } \\
\text { l/100km }\end{array}$ \\
\hline $\mathbf{P E}$ & 17133.2 & 10.53 & 182.09 & 3.73 \\
$\mathbf{C I}$ & 15758.9 & 13.48 & 162.42 & 4.00 \\
$\mathbf{F I}$ & 15257.2 & 10.97 & 172.45 & 4.26 \\
$\mathbf{S K}$ & 15139.3 & 14.12 & 170.71 & 3.99 \\
$\mathbf{L A}$ & 15865.3 & 11.76 & 183.10 & 3.41 \\
\hline & & & & \\
\hline $\mathbf{q}_{\mathbf{j}}$ & 783.50 & 2.52 & 30 & 0.24 \\
$\mathbf{p}_{\mathbf{j}}$ & 1757.29 & 4.08 & 40 & 0.5 \\
\hline
\end{tabular}

Inspired by Lahdelma and Salminen (2002a), the analyst could be interested in understanding whether the results provided by the method are dependent on the values assigned to the considered thresholds. For this reason, we applied SMAA to 2000 randomly generated problems. Each problem was composed as follow. We sampled a performance table $M$ and a vector of weights $w$ compatible with the preference information provided by the DM. Then, by considering $M$ and $w$, we sampled 10000 thresholds in the considered intervals and we studied, consequently, the rank acceptability indices obtained by applying SMAA.

Table 7: Robustness analysis on indifference and preference thresholds

\begin{tabular}{cc}
\hline Number of alternatives having $b_{j}^{1}>0$ & Problems \\
\hline 1 & 1501 \\
2 & 422 \\
4 & 69 \\
5 & 8 \\
\hline
\end{tabular}


Looking at Table 7, we could state that, fixing the performance table and the weight vector, in same cases the variation of the thresholds does not influence the results of the model while in all others cases it could marginally influence the results. On one side, out of 1501 problems, only one alternative had $b_{k}^{1}>0$ after applying SMAA. This means that only one alternative can be ranked first rank whilst also changing the thresholds. On the other side, in no problem all alternatives can have $b_{k}^{1}>0$ whereas out of 8 problems only four out of the five considered alternatives can fill the first position (having $b_{k}^{1}>0$ ). This means that, in this example, the variation of the thresholds won't highly influence the results obtained by applying SMAA once that the performance table and the criteria weights have already been fixed.

In general, we could not state that the values given to the thresholds are not influencing the results but that the DM should perform in any case a sensitivity analysis to test the robustness of the obtained results.

\section{Conclusions}

PROMETHEE methods and SMAA have been widely applied to deal with several real world problems (Behzadian et al., 2010; Tervonen and Figueira, 2008). In this paper we have proposed to apply SMAA to the classical PROMETHEE methods (Brans and Mareschal, 2005; Brans et al., 1984). The integration of SMAA and PROMETHEE methods gives the opportunity to obtain recommendations considering several set of parameters compatible with the preference information provided by the DM, and not only considering one of these sets. Thus the methodology presented in this paper brings about the possibility to effectively deal with robustness concerns related to the choice of preference parameters in PROMETHEE methods.

In the PROMETHEE II case, one can look at the possible final rankings obtained considering the net flow and at which parameters give to an alternative the best position.

In the didactic example we have shown that the SMAA methodology can be applied to classical PROMETHEE methods. This brings us to believe that the methodology we are proposing in this paper can be adopted to deal with many real-world problems.

In the future we also plan on applying the SMAA methodology to the bipolar PROMETHEE methods (Corrente et al., 2012) being the extension of the classic PROMETHEE methods to the case of interacting criteria and to the MCHP applied to PROMETHEE methods (Corrente et al., 2013).

\section{Acknowledgments}

The authors would like to thank the the comments and suggestions of the three anonymous reviewers which have helped to considerably improve this manuscript. 


\section{References}

W. Aertens, V. Kint, J. Van Orshoven, and B. Muys. Evaluation of modelling techniques for forest site productivity prediction in contrasting ecoregions using stochastic multicriteria acceptability analysis (SMAA). Environmental Modelling \& Software, 26(7):929-937, 2011.

S. Angilella, S. Greco, and B. Matarazzo. Non-additive robust ordinal regression: A multiple criteria decision model based on the Choquet integral. European Journal of Operational Research, 201(1):277-288, 2010.

S. Angilella, S. Corrente, and S. Greco. SMAA-Choquet: Stochastic Multicriteria Acceptability Analysis for the Choquet Integral. In S. Greco et al., editor, IPMU 2012, Part IV, CCIS 300, pages 248-257. Springer-Verlag Berlin Heidelberg 2012, 2012.

M. Behzadian, R.B. Kazemzadeh, A. Albadvi, and M. Aghdasi. PROMETHEE: A comprehensive literature review on methodologies and applications. European Journal of Operational Research, 200(1):198-215, 2010.

J.P. Brans and B. Mareschal. PROMETHEE Methods. In J. Figueira, S. Greco, and M. Ehrgott, editors, Multiple Criteria Decision Analysis: State of the Art Surveys, pages 163-196. Springer, Berlin, 2005.

J.P. Brans and Ph. Vincke. A preference ranking organisation method: The PROMETHEE method for MCDM. Management Science, 31(6):647-656, 1985.

J.P. Brans, B. Mareschal, and Ph. Vincke. PROMETHEE: a new family of outranking methods in multicriteria analysis. In J.P. Brans, editor, Operational Research, IFORS 84, pages 477-490. North Holland, Amsterdam, 1984.

E. Carrizosa and F.R. Puerto J. Conde, E. Fernandez. Multi-criteria analysis with partial information about the weighting coefficients. European Journal of Operational Redearch, 81:291-301, 1995.

M.N.A.L. Claessens, F.A. Lootsma, and F.J. Vogt. An elementary proof of Paelinck's theorem on the convex hull of ranked criterion weights. European Journal of Operational Research, 52(2):255-258, 1991.

S. Corrente, J.R. Figueira, and S. Greco. Interaction of criteria and robust ordinal regression in bi-polar PROMETHEE methods. In S. Greco et al., editor, IPMU 2012, Part IV, CCIS 300, pages 469-479. Springer, Berlin, 2012.

S. Corrente, S. Greco, and R. Słowiński. Multiple Criteria Hierarchy Process with ELECTRE and PROMETHEE. Omega, 41(5):820-846, 2013. 
S. Corrente, J.R. Figueira, and S. Greco. Dealing with Interaction Between Bipolar Multiple Criteria Preferences in PROMETHEE Methods. Annals of Operational Research, 217(1):137-164, 2014.

L.C. Dias and V. Mousseau. Inferring ELECTRE's veto-related parameters from outranking examples. European Journal of Operational Research, 170(1):172-191, 2006.

I. Durbach. On the estimation of a satisficing model of choice using stochastic multicriteria acceptability analysis. Omega, 37(3):497-509, 2009.

S. Eppe and Y. De Smet. Studying the Impact of Information Structure in the PROMETHEE II Preference Elicitation Process: A Simulation Based Approach. In S. Greco et al., editor, IPMU 2012, Part IV, CCIS 300, pages 383-392. Springer-Verlag Berlin Heidelberg 2012, 2012.

S. Eppe, Y. De Smet, and T. Stutzle. A Bi-Objective Optimization Model to Eliciting Decision Maker's Preferences for the PROMETHEE II Method. In R.I. Brafman, F. Roberts, and A. Tsoukias, editors, ADT 2011. LNCD, vol. 6992, pages 56-66. Springer Heidelberg 2011, 2011.

J. Figueira, S. Greco, and M. Ehrgott (eds.). Multiple Criteria Decision Analysis: State of the Art Surveys. Springer, Berlin, 2005a.

J. Figueira, V. Mousseau, and B. Roy. ELECTRE methods. In J. Figueira, S. Greco, and M. Ehrgott, editors, Multiple Criteria Decision Analysis: State of the Art Surveys, pages 133-153. Springer, Berlin, 2005b.

A. Giarlotta and S. Greco. Necessary and possible preference structures. Journal of Mathematical Economics, 49(2):163-172, 2013.

S. Greco, V. Mousseau, and R. Słowiński. Ordinal regression revisited: multiple criteria ranking using a set of additive value functions. European Journal of Operational Research, 191(2):416-436, 2008.

S. Greco, R. Słowiński, J.R. Figueira, and V. Mousseau. Robust ordinal regression. In M. Ehrgott, J. Figueira, and S. Greco, editors, Trends in Multiple Criteria Decision Analysis, pages 273-320. Springer, Berlin, 2010.

S. Greco, M. Kadziński, V. Mousseau, and R. Słowiński. ELECTRE ${ }^{G K M S}$ : Robust ordinal regression for outranking methods. European Journal of Operational Research, 214(1):118-135, 2011.

J. Hokkanen, R. Lahdelma, K. Miettinen, and P. Salminen. Determining the implementation order of a general plan by using a multicriteria method. Journal of Multi-Criteria Decision Analysis, 7(5):273-284, 1998. 
M. Kadziński and T. Tervonen. Robust multi-criteria ranking with additive value models and holistic pair-wise preference statements. European Journal of Operational Research, 228(1):169-180, 2013a.

M. Kadziński and T. Tervonen. Stochastic ordinal regression for multiple criteria sorting problems. Decision Support Systems, 55(11):55-66, 2013b.

M. Kadziński, S. Greco, and R. Słowiński. Extreme ranking analysis in robust ordinal regression. Omega, 40(4):488-501, 2012.

R. Lahdelma and P. Salminen. SMAA-2: Stochastic multicriteria acceptability analysis for group decision making. Operations Research, 49(3):444-454, 2001.

R. Lahdelma and P. Salminen. Pseudo-criteria versus linear utility function in stochastic multi-criteria acceptability analysis. European Journal of Operational Research, 141(2):454-469, 2002a.

R. Lahdelma and P. Salminen. Modelling dependent uncertainties by multivariate gaussian distributions in smaa. Technical Report 471, 2002b.

R. Lahdelma and P. Salminen. Prospect theory and stochastic acceptability analysis (SMAA). Omega, 37 (5):961-971, 2009.

R. Lahdelma, J. Hokkanen, and P. Salminen. SMAA - Stochastic multiobjective acceptability analysis. European Journal of Operational Research, 106(1):137-143, 1998.

R. Lahdelma, K. Miettinen, and P. Salminen. Ordinal criteria in stochastic multicriteria acceptability analysis (SMAA). European Journal of Operational Research, 147(1):117-127, 2003.

R. Lahdelma, S. Makkonen, and P. Salminen. Multivariate Gaussian criteria in SMAA. European Journal of Operational Research, 170(3):957-970, 2006.

P. Leskinen, J. Viitanen, A. Kangas, and J. Kangas. Alternatives to incorporate uncertainty and risk attitude in multicriteria evaluation of forest plans. Forest Science, 52(3):304-312, 2006.

B. Mareschal. Weight stability intervals in multicriteria decision aid. European Journal of Operational Research, 33(1):54-64, 1988.

A.M. Mármol, J. Puerto, and F.R. Fernández. The Use of Partial Information on Weigths in Multicriteria Decision Problems. Journal of Multi-criteria decision analysis, 7:322-329, 1998.

A. Menou, A. Benallou, R. Lahdelma, and P. Salminen. Decision support for centralizing cargo at a Moroccan airport hub using stochastic multicriteria acceptability analysis. European Journal of Operational Research, 204(3):621-629, 2010. 
V. Mousseau and R. Słowiński. Inferring an ELECTRE TRI model from assignment examples. Journal of Global Optimization, 12(2):157-174, 1998.

V. Mousseau, R. Słowiński, and P. Zielniewicz. A user-oriented implementation of the ELECTRE-TRI method integrating preference elicitation support. Computers $\&$ Operations Research, 27(7-8):757-777, 2000 .

J.H.P. Paelinck. Qualitative multiple-criteria analysis, environmental protection and multiregional development. Papers of the Regional Science Association, 36:59-74, 1974.

J. Puerto, A.M. Mármol, L. Monroy, and F.R. Fernández. Decision criteria with partial information. International Transactions in Operational Research, 7:51-65, 2000.

R.L Smith. Efficient Monte Carlo procedures for generating points uniformly distributed over bounded regions. Operations Research, 32:1296-1308, 1984.

T. Solymosi and J. Dombi. A method for determining the weights of criteria: the centralized weights. European Journal of Operational Research, 26(1):35-41, 1986.

Z. Sun and M. Han. Multi-criteria decision making based on PROMETHEE method. In Proceedings of the 2010 International Conference on Computing Control and Industrial Engineering, IEEE Computer Society Press, pages 416-418, 2010.

T. Tervonen and J. Figueira. A survey on stochastic multicriteria acceptability analysis methods. Journal of Multi-Criteria Decision Analysis, 15(1-2):1-14, 2008.

T. Tervonen and R. Lahdelma. Implementing stochastic multicriteria acceptability analysis. European Journal of Operational Research, 178(2):500-513, 2007.

T. Tervonen, J. Figueira, R. Lahdelma, J. Almeida Dias, and P. Salminen. A stochastic method for robustness analysis in sorting problems. European Journal of Operational Research, 192(1):236-242, 2009a.

T Tervonen, J. Figueira, R Lahdelma, and P Salminen. SMAA-III: A simulation-based approach for sensitivity analysis of ELECTRE III. In Real-Time and Deliberative Decision Making, pages 241-253. Springer, 2009b.

T. Tervonen, I. Linkov, J. R. Figueira, J. Steevens, M. Chappel, and M. Merad. Risk-based classification system of nanomaterials. Journal of Nanoparticle Research, 11(4):757-766, 2009c. 
T. Tervonen, G. Van Valkenhoef, N. Basturk, and D. Postmus. Hit-and-run enables efficient weight generation for simulation-based multiple criteria decision analysis. European Journal of Operational Research, 224:552-559, 2013.

W.T.M. Wolters and B. Mareschal. Novel types of sensitivity analysis for additive MCDM methods. European Journal of Operational Research, 81(2):281-290, 1995. 